\title{
Stalled Progress: Medical School Dean Demographics
}

\author{
Autumn Nobles, BA, Bianka Aceves Martin, BS, Jaileessa Casimir, BS, \\ Sarah Schmitt, BS, and Geoffrey Broadbent, BS
}

Medical schools have an important directive: to train the next generation of physicians. Faced with a primary care physician shortage, increasing numbers of under-represented faculty leaving academic medicine, low representation of women in leadership positions, and an ongoing pandemic, medical schools have a duty to implement solutions to alleviate these issues. Efforts have been made to create more diverse medical school classes, but those efforts are not mirrored in senior faculty demographics. In this medical students' perspective piece, the authors analyzed the demographics of medical school deans in comparison with the United States' demographics and the current composition of active physicians. The authors looked at the specialty, race/ethnicity, and gender of medical school deans in 2019. Based on the analysis, in 2019 only $11 \%$ of deans were under-represented minorities, $16 \%$ of deans were primary care physicians, and $18 \%$ of deans were women. When compared with the makeup of physicians in the United States and the population as a whole, these numbers are unrepresentative of national demographics. By hiring deans with a variety of race/ ethnicities, specialties, and genders, schools set an important precedent that could lead to more pipeline programs, increased under-represented faculty retention, and more primary care physicians. (J Am Board Fam Med 2022;35:163-168.)

Keywords: Career Choice, Faculty, Family Medicine, Leadership, Medical Schools, Minority Groups, Primary Care Physicians, Primary Health Care, Under-represented in medicine

The COVID-19 pandemic has exacerbated and uncovered the health disparities present in our communities and has increased the push for health equity across the country. ${ }^{1,2}$ This has led to a movement to examine and find solutions for the health care system's role in perpetuating these health inequities in communities of color. ${ }^{3}$ Two essential ways to improve health equity are to increase the diversity of physicians ${ }^{4,5}$ and move health care upstream to focus on prevention and primary care. ${ }^{6}$

This article was externally peer reviewed.

Submitted 19 April 2021; revised 8 July 2021; accepted 13 July 2021.

From Yale School of Medicine, New Haven, CT (AN); University of California Riverside School of Medicine, Riverside, CA (BAM); City University of New York School of Medicine, New York, NY (JC); George Washington University School of Medicine and Health Sciences, Washington, DC (SS, GB).

Funding: none.

Conflict of interest: none.

Corresponding author: Autumn Nobles, BA, 524 George St., New Haven, CT, 06511 (E-mail: autumn.nobles@yale. edu).
With a majority-minority nation on the horizon for 2044, the need for physicians who resemble and represent our population demographics is increasingly important. ${ }^{7}$ Studies have shown that medical school compositional diversity increases medical students' cultural awareness, ${ }^{8}$ decreases implicit bias, ${ }^{8}$ and encourages positive attitudes toward diversity. ${ }^{9,10}$ Hospitals with higher proportions of racial and ethnic minorities in senior leadership positions have more initiatives that aim to achieve health equity and reduce disparities. ${ }^{11}$

Historically, minority populations have been restricted from opportunities and promotions in their medical training, memberships, and professional activities, which subsequently excluded them from participating and impacting decisions at the institutional level. ${ }^{12,13}$ There are various factors that perpetuate institutional racism and ostracize patients, students, physicians, and leaders from these minority communities. In medical education, such factors include lack of access to medical education for those under-represented in medicine (URM) and other neglected groups, ${ }^{14}$ medical student experiences of 
discrimination and bias, ${ }^{15}$ and minority faculty experiences of the "minority tax," the increased burden placed on minority faculty to lead diversity initiatives. $^{16,17}$ Historically Black Colleges and Universities (HBCUs) are leaders and innovators in supporting pipeline programs built to expose URMs to the health professions ${ }^{18}$ and tend to produce large numbers of minority medical school applicants as well as minority medical school deans across the United States. ${ }^{19}$ This trend continues even into medical school, with $20 \%$ of Black matriculants attending the 4 HBCUs with medical schools. ${ }^{20}$ However, it is imperative that the task of diversifying medical education is not solely driven by HBCUs. Diversity must be actively embraced by all institutions, especially predominately White institutions.

Minority physicians are also important in solving the issues revolving around the shortage of primary care physicians. Black and African American physicians do not account for the majority of active physicians in the United States, yet a plurality of Black or African American physicians practiced primary care ( $41.4 \%$ vs $30.6 \%$ of White physicians) in $2018 .^{21}$ Minority physicians are also more likely to work in medically underserved areas or in health professions shortage areas. ${ }^{22}$ One study found that $15 \%$ of White physicians practiced in a Medically Underserved Area, compared with 28\% of African American physicians, 24\% of Latino physicians, and $19 \%$ of Asian physicians. ${ }^{23}$

Primary care physicians play an essential role in the prevention of hospitalizations and the management of chronic conditions, and evidence demonstrates more primary care physicians are associated with better health outcomes. ${ }^{6,24}$ Despite this evidence, it is estimated that by 2025 there will be a deficit of up to 31,000 adult primary care physicians in the United States. ${ }^{25}$ When examining the causes of this shortage, studies have suggested students do not choose primary care due to lack of role models in primary care, burnout associated with addressing the social needs of underserved populations, and professional cultures that view primary care negatively. ${ }^{26,27}$ Deans and administrators are important in shaping the culture in medical schools and can help eliminate (or exacerbate) stigma against primary care. ${ }^{27}$

With women now making up over half of medical school matriculants, the medical leadership gap can no longer be attributed to the number of women choosing medicine. Nevertheless, women are still under-represented in senior leadership ranks and department chairs. ${ }^{28}$ Seeing this representation matters. Students who identified as female had higher odds of reporting that faculty lacked respect for diversity when compared with males. ${ }^{29}$ Previous research has demonstrated that gender-based discrimination is still prevalent in medical education, and women are sorely needed in these spaces. ${ }^{28}$

Senior leadership can help bring these muchneeded changes to medical schools. ${ }^{30}$ Leaders can bring in new perspectives and prioritize building a diverse and knowledgeable community. These changes might include structural changes, such as creating pipeline programs and re-evaluating admissions policies, as well as curricular changes, such as promoting community-based training, health equity curriculum, and removing content that perpetuates the myth of biological race. The aforementioned changes have been implemented in several medical schools, including the Medical University of South Carolina, with successful programs dedicated to increasing diversity. To enumerate certain critical elements, they focused on: (1) fostering an institutional culture that embraces diversity, (2) demonstrating a commitment to diversity within institutional leadership, (3) developing admission policies to promote diversity, and (4) clearly articulated institutional policies that uphold a culture supportive of diversity. ${ }^{31}$ Increasing primary care physician representation in senior leadership would convey to students that a career in primary care is respected and a path to future professional success. For students, diversity at the highest levels is an important signal of a school's priorities and culture and provides role models for future careers. This is evidenced by HBCU medical schools, headed by minority deans, leading medical schools in producing graduates that are URM, going into primary care, and pursuing work in underserved areas. ${ }^{32,33}$

Amid the Black Lives Matter movement of the past year, many medical schools released statements on the value of a diverse representation of race, ethnicity, and gender in their schools and senior leadership. However, the ongoing calls for more diverse leadership demonstrate that this value is not being fully realized. ${ }^{34,35}$ Leadership roles need to be filled by individuals of different races, ethnicities, genders, and specialties to implement changes needed to strive toward health equity. ${ }^{31}$ 
Figure 1. US medical schools deans race/ethnicity, 1966 to 2019. Source: AAMC Council of Deans records, as of 8/17/2020; U.S. population demographics from U.S. Census Bureau. Abbreviation: URM, under-represented in medicine.

URM Medical School Deans, 1966-2019

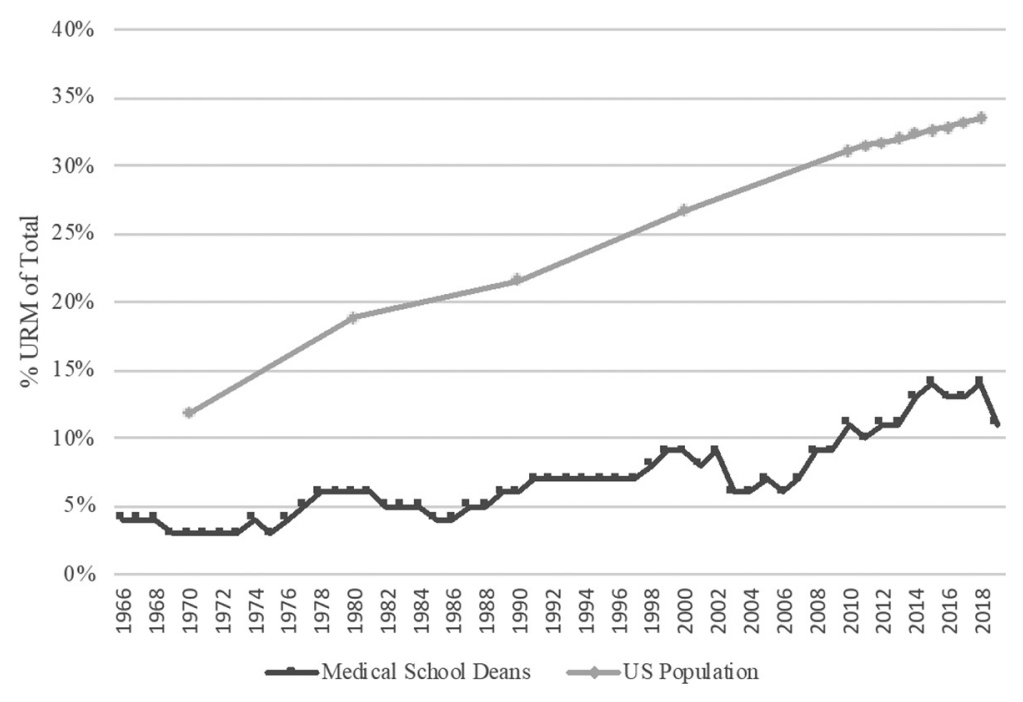

\section{Medical School Deans in 2019}

To shed light on these issues of representation, we examined the specialty, gender, and race/ethnicity of 153 allopathic medical school deans. Information regarding the gender of medical school deans is publicly available online by the Association of American Medical Colleges (AAMC). We further obtained data on the race/ethnicity of deans from the AAMC, which they collect through their AAMC Council of Deans. We focused on the data contained in the AAMC report, which excluded osteopathic deans. We determined the medical specialties of deans in 2019 by reviewing their online biographies or dean's announcements.

Based on our findings, as medical students and future physicians, we are deeply concerned with the lack of diversity among the highest level of leadership within our medical education communities.

While the number of women deans has increased since 1990, when women represented just $1 \%$ of deans, ${ }^{36}$ in 2019 , women continue to be severely under-represented at just $18 \%$ of medical school deans. Women have made strides in terms of representation within the overall medical community; however, this has not translated to the leadership level at medical schools. We need more women in higher positions of power in medical schools across the country.

In $2019,11 \%$ of deans were considered to be URM, compared with $33 \%$ in the US population.
The AAMC defines URM as those "racial and ethnic populations that are under-represented in the medical profession relative to their numbers in the general population." ${ }^{37}$ This lack of diversity has been present since data were first collected in the 1970s, when the percentage of URM among medical school deans was $4 \%$, compared with $12 \%$ of the general population. Even as late as the early 2000 s, only around $9 \%$ of deans were URM, compared with $27 \%$ of the population (Figure 1). Despite these increases, the rate of the diversifications of deans is not proportional to that of the nation, as seen in Figure 1. The $8 \mathrm{HBCU}$ and Puerto Rican medical schools from our analysis disproportionately contribute to URM representation among medical school deans. For instance, in 2019, they contributed almost half of the 17 URM-identifying deans.

We found that only $16 \%$ of deans were from primary care fields, while $32.1 \%$ of active physicians practice in primary care. ${ }^{21}$ Specialties we categorized as primary care included family medicine, internal medicine, and pediatrics, with no further specialization.

\section{A Road Map Forward}

The diversity of senior leadership matters to students. ${ }^{29}$ Medical schools should hire deans and senior leadership that represent a diversity of 
specialties, genders, races, and ethnicities. Future physicians need to acquire skills to care for increasingly diverse groups by learning about the unique needs of minority populations and by including social determinants of health, cultural humility, and systemic racism in medical curricula. ${ }^{38-41}$ Schools should prioritize issues that matter to their primary constituents- - their students. ${ }^{38}$

While the medical school enrollment gender gap has been closed, the number of women in senior positions lags behind. ${ }^{42}$ When implemented, evidence-based practices that use targeted mentorship and career development programs, education of search committees, and institutional accountability measures increase the number of women in faculty positions. ${ }^{43-47}$ However, current uptake of these methods is scattered and typically limited to individual institutions.

The number of under-represented minorities in medicine continues to trail behind general population numbers, with research showing it would take nearly 1000 years for the numbers of Black physicians to catch up. ${ }^{48} \mathrm{We}$ must create a positive cultural change to support URM students and their aspirations and the retention and promotion of more URM faculty through increased support for pipeline programs as well as mentorship and faculty development for junior faculty. ${ }^{49-51}$ We need URM leaders in the highest level within all medical schools to serve as role models for success, not just at HBCUs and in Puerto Rico. ${ }^{52,53}$

While the choice of primary care careers is also driven by factors such as income and practice challenges, the number of deans from primary care fields remains unrepresentative. Given the importance of primary care in the prevention and maintenance of health, as well as the growing primary care shortage, we believe that more primary care deans are needed. Institutional culture affects how students perceive different specialties, and primary care physicians in leadership positions will positively change this culture. ${ }^{27}$ More programs such as the Society of Teachers of Family Medicine's Bishop Fellowship program that provides training and support for family medicine faculty looking for senior positions, including access to a dean for 3 weeks, could help family medicine faculty to take up these roles. ${ }^{5,54}$ Deans who represent these fields serve as an inspiration to students and show that leadership in medicine need not be limited to specialty practitioners alone. It also demonstrates that promising future careers await those who pursue these critically needed specialties.

Based on our findings around the lack of representation of women, URMs, and primary care physicians as deans, medical schools should increase efforts to develop faculty that identify with these groups. These changes could be achieved through pipeline programs, increased focus on the mentoring and career development of URMs and women, and continued mentorship for those interested in primary care. These adjustments would help make medicine more inclusive and representative, not only for the next generation of physicians but also for the populations we serve.

An eternal thank you to the Beyond Flexner Alliance, Candice Chen, and Jamar Slocum for funding, editing, and supporting this work.

To see this article online, please go to: http://jabfm.org/content/ 35/1/163.full.

\section{References}

1. Racial and ethnic disparities in COVID-19-related infections, hospitalizations, and deaths: a systematic review [Internet]. Ann Intern Med; 2021 [cited 2021 June 9]. Available from: https://www. acpjournals.org/doi/full/10.7326/M20-6306.

2. Bibbins-Domingo $\mathrm{K}$. This time must be different: disparities during the COVID-19 pandemic. Ann Intern Med 2020;173:233-4.

3. Razai MS, Kankam HKN, Majeed A, Esmail A, Williams DR. Mitigating ethnic disparities in COVID-19 and beyond. BMJ 2021;372:m4921.

4. Cohen JJ, Gabriel BA, Terrell C. The case for diversity in the health care workforce. Health Aff (Millwood) 2002;21:90-102.

5. National Academies of Science, Engineering, Medicine. Implementing high-quality primary care: rebuilding the foundation of health care. Washington (DC): National Academies Press; 2021.

6. Contribution of primary care to health systems and health [Internet]. Milbank Quarterly; 2005 [cited 2021 Jan 11]. Available from: https://onlinelibrary. wiley.com/doi/full/10.1111/j.1468-0009.2005.00409.x.

7. Colby S, Ortman JM [Internet]. Projections of the size and composition of the U.S. population: 2014 to $2060 ; 2015: 13$. Available from: https://www. census.gov/library/publications/2015/demo/p25-1143. html.

8. Saha S, Guiton G, Wimmers PF, Wilkerson L. Student body racial and ethnic composition and diversity-related outcomes in US medical schools. JAMA 2008;300:1135-45.

9. Guiton G, Chang MJ, Wilkerson L. Student body diversity: relationship to medical students' experiences and attitudes. Acad Med J Med 2007;82:S85-88. 
10. Association between perceived medical school diversity climate and change in depressive symptoms among medical students: a report from the medical student CHANGE study [Internet]. ClinicalKey; 2016 [cited 2021 June 13]. Available from: https:// www.clinicalkey.com/\#!/content/playContent/1-s2.0S0027968416301018? returnurl=https:\%2F\%2Flin kinghub.elsevier.com \%2Fretrieve $\% 2$ Fpii $\% 2 F S 00$ $27968416301018 \% 3$ Fshowall\%3Dtrue\&referrer= https:\%2F\%2Fjamanetwork.com $\% 2 \mathrm{~F}$.

11. Herrin J, Harris KG, Spatz E, Cobbs-Lomax D, Allen S, León T. Hospital leadership diversity and strategies to advance health equity. Jt Comm J Qual Patient Saf 2018;44:545-51.

12. Smedley BD, Butler AS, Bristow LR [Internet]. Increasing diversity in the health professions: a look at best practices in admissions. National Academies Press; 2004 [cited 2021 June 9]. Available from:. https://www.ncbi.nlm.nih.gov/books/NBK216007/.

13. Boatright D, O'Connor PG, E Miller J. Racial privilege and medical student awards: addressing racial disparities in Alpha Omega Alpha honor society membership. J Gen Intern Med 2020;35: 3348-51.

14. Campbell KM, Brownstein NC, Livingston H, Rodríguez JE. Improving underrepresented minority in medicine representation in medical school. South Med J 2018;111:203-8.

15. Hill KA, Samuels EA, Gross CP, et al. Assessment of the prevalence of medical student mistreatment by sex, race/ethnicity, and sexual orientation. JAMA Intern Med 2020;180:653-65.

16. Mahoney MR, Wilson E, Odom KL, Flowers L, Adler SR. Minority faculty voices on diversity in academic medicine: perspectives from one school. Acad Med J Med 2008;83:781-6.

17. Rodríguez JE, Campbell KM, Pololi LH. Addressing disparities in academic medicine: what of the minority tax? BMC Med Educ 2015;15:6.

18. Smith SG, Nsiah-Kumi PA, Jones PR, Pamies RJ. Pipeline programs in the health professions, part 1: preserving diversity and reducing health disparities. J Natl Med Assoc 2009;101:836-51.

19. Hoover E. An analysis of the Association of American Medical Colleges' review of minorities in medical education. J Natl Med Assoc 2005;97: 1240-56.

20. Rodríguez JE, López IA, Campbell KM, Dutton M. The role of Historically Black College and University medical schools in academic medicine. J Health Care Poor Underserved 2017;28: 266-78.

21. Association of American Medical Colleges [Internet]. Diversity in medicine: facts and figures 2019; 2021 [cited 2021 June 16]. Available from: https://www.aamc.org/data-reports/workforce/ interactive-data/figure-26-primary-care-versusnonprimary-care-physicians-race/ethnicity-2018.
22. Goodfellow A, Ulloa JG, Dowling PT, et al. Predictors of primary care physician practice location in underserved urban or rural areas in the United States: a systematic literature review. Acad Med 2016;91:1313-21.

23. Walker KO, Moreno G, Grumbach K. The association among specialty, race, ethnicity, and practice location among California physicians in diverse specialties. J Natl Med Assoc 2012;104:46-52.

24. Basu S, Berkowitz SA, Phillips RL, Bitton A, Landon BE, Phillips RS. Association of primary care physician supply with population mortality in the United States, 2005-2015. JAMA Intern Med 2019;179:506-14.

25. U.S. Department of Health and Human Services, Health Resources and Services Administration, National Center for Health Workforce Analysis [Internet]. National and regional projections of supply and demand for primary care practitioners: 2013-2025; 2016. Available from: https://bhw.hrsa. gov/sites/default/files/bureau-health-workforce/ training/projections-2025.pdf.

26. Long T, Chaiyachati K, Bosu O, et al. Why aren't more primary care residents going into primary care? A qualitative study. J Gen Intern Med 2016;31:1452-9.

27. Erikson CE, Danish S, Jones KC, Sandberg SF, Carle AC. The role of medical school culture in primary care career choice. Acad Med 2013;88:1919-26.

28. 2018-2019 the state of women in academic medicine: exploring pathways to equity [Internet]. AAMC; 2021 [cited 2021 June 13]. Available from: https://www.aamc.org/data-reports/data/2018-2019state-women-academic-medicine-exploring-pathwaysequity.

29. Weiss J, Balasuriya L, Cramer LD, et al. Medical students' demographic characteristics and their perceptions of faculty role modeling of respect for diversity. JAMA Netw Open 2021;4:e2112795.

30. Hromas R, Leverence R, Mramba LK, et al. What a medical school chair wants from the dean. J Healthc Leadersh 2018;10:33-44.

31. Deas D, Pisano ED, Mainous AG, et al. Improving diversity through strategic planning: a 10-year (2002-2012) experience at the Medical University of South Carolina. Acad Med J Med 2012;87: 1548-55.

32. Mullan F, Chen C, Petterson S, Kolsky G, Spagnola M [Internet]. The social mission of medical education: ranking the schools. Ann Intern Med; 2010 [cited 2021 June 13]. Available from: https:// www.acpjournals.org/doi/abs/10.7326/0003-4819152-12-201006150-00009.

33. Ko M, Heslin KC, Edelstein RA, Grumbach K. The role of medical education in reducing health care disparities: the first ten years of the UCLA/ Drew Medical Education Program. J Gen Intern Med 2007;22:625-31. 
34. Acosta DA, Poll-Hunter NI. Diversity and inclusion in the academic medicine workforce. In: Sánchez JP, editor. Succeeding in academic medicine: a roadmap for diverse medical students and residents. New York: Springer; 2020. p. 1-14.

35. Coe C, Piggott C, Davis A, et al. Leadership pathways in academic family medicine: focus on underrepresented minorities and women. Fam Med 2020;52:104-11.

36. Association of American Medical Colleges [Internet]. U.S. medical school deans by dean type and sex; 2021 [cited 2021 Sept 15]. Available from: https://www.aamc.org/data-reports/facultyinstitutions/interactive-data/us-medical-schooldeans-dean-type-and-sex.

37. Underrepresented in medicine definition [Internet]. AAMC; 2021 [cited 2021 June 7]. Available from: https://www.aamc.org/what-we-do/equity-diversityinclusion/underrepresented-in-medicine.

38. Shahriar AA, Prasad K, Casty K, Rahman ZI, Westerhaus M, Satin DJ. Race and gender differences in medical student perspectives on social determinants of health education: a single-institution survey study. Adv Med Educ Pract 2021;12:587-95.

39. Learning objectives for medical student education-guidelines for medical schools: report I of the Medical School Objectives Project [Internet]. Academic Medicine; 2021 [cited 2021 June 13]. Available from: https://journals.lww.com/academic medicine/Abstract/1999/01000/Learning_Objectives_ for_Medical_Student_Education_.10.aspx.

40. Social justice should be a key part of educating health professionals [Internet]. STAT; 2017 [cited 2021 June 13]. Available from: https://www. statnews.com/2017/04/07/social-justice-healtheducation/.

41. Gruen RL, Pearson SD, Brennan TA. Physiciancitizens-public roles and professional obligations. JAMA 2004;291:94-8.

42. AAFP [Internet]. Women have closed med school enrollment gap; others remain; 2020 [cited 2021 June 13]. Available from: https://www.aafp.org/ news/blogs/leadervoices/entry/20200228lv-diversity. html.

43. Valantine HA, Grewal D, Ku MC, et al. The gender gap in academic medicine: comparing results from a multifaceted intervention for Stanford faculty to peer and national cohorts. Acad Med 2014;89:904-11.

44. Stewart AJ, Vaque-Manty DL, Malley JE. Recruiting female faculty members in science and engineering: preliminary evaluation of one intervention model. J Women Minor Sci Eng 2004;10:50.

45. Sheridan JT, Fine E, Pribbenow CM, Handelsman J, Carnes M. Searching for excellence \& diversity: increasing the hiring of women faculty at one academic medical center. Acad Med 2010;85:999-1007.

46. Career development for women in academic medicine: multiple interventions in a department of medicine [Internet]. JAMA; 2021 [cited 2021 June 13]. Available from: https://jamanetwork.com/ journals/jama/article-abstract/407999.

47. Kalev A, Dobbin F, Kelly E. Best practices or best guesses? Assessing the efficacy of corporate affirmative action and diversity policies. Am Sociol Rev 2006;71:589-617.

48. Yu PT, Parsa PV, Hassanein O, Rogers SO, Chang DC. Minorities struggle to advance in academic medicine: a 12-y review of diversity at the highest levels of America's teaching institutions. J Surg Res 2013;182:212-8.

49. Clayborne EP, Martin DR, Goett RR, Chandrasekaran EB, McGreevy J. Diversity pipelines: the rationale to recruit and support minority physicians. J Am Coll Emerg Physicians Open 2021;2:e12343.

50. Doll KM, Thomas CR. Structural solutions for the rarest of the rare-underrepresented-minority faculty in medical subspecialties. $\mathrm{N}$ Engl J Med 2020;383:283-5.

51. Rodriguez JE, Campbell KM, Fogarty JP, Williams RL. Underrepresented minority faculty in academic medicine: a systematic review of URM faculty development. Fam Med 2014;46:100-4.

52. Gasman M, Smith T, Ye C, Nguyen T-H. HBCUs and the production of doctors. AIMS Public Health 2017;4:579-89.

53. Lakhan SE, Laird C. Addressing the primary care physician shortage in an evolving medical workforce. Int Arch Med 2009;2:14.

54. Dickson G. Leading family medicine into the future: are we prepared? Ann Fam Med 2011;9: 465-6. 